\title{
Implementing Multiprotocol Label Switching Technology for Secure Network Communication between Organizations
}

\author{
Hamed Abdullah Hamed Al Bulushi ${ }^{\mathrm{a}}$, and Indrani Palanisamy ${ }^{\mathrm{a}}$
}

\begin{abstract}
This exploratory study analysis the current complications in the Manufacturing organizations for transferring files between two or more sites in-between an organization over an unsecure network communication. Based on the analysis we provide reliable implementation by an appropriate solution to improve and develop the performance of the network communication to help the company to progress the quality of work. The initial phase of the study analyses about Virtual Private Network (VPN) based on the network layers and identifies the underneath difficulties in VPN while data travels over the network in the form of encapsulation and the traffic of the fundamental network. Because of such encapsulation technique used in VPN it increases the network traffic which affects the bandwidth of a low speed internetworking communication. By analyzing various case studies, it was identified Multiprotocol Label Switching (MPLS) technology gives a feasible solution to put the network communication on the right track. It creates secure and reliable connectivity between organization sites and was effectively implemented in the place where slow speed network for connectivity between organizations are found. The recommending this MPLS solution, it helps organizations to share and effectively utilize the servers and service between organizations for secure, reliable and effective communication. Also by considering network security factor as a very significant factor for any network, we have also implemented the Firewall which provide appropriate defense for networks that prevents from internal and external threats. At same time the solution also provides support to Wireless LAN Controller (WLC) for providing easy manage and control all wireless access point.
\end{abstract}

Keywords: Firewall, Multi-protocol Label Switching (MPLS), Servers, Virtual Private Network (VPN), Wireless LAN Controller (WLC).

\section{Introduction}

Multiprotocol Label Switching (MPLS) is a technique that carry a data for high performance telecom networks from one network to another by using shortest path, also has stable and scalable line which able to build new servers and expand the company new branches. Depends on the existing company network issues, this project will help to enhance the existing company network which its direct impact in improving the quality of work, fast communication between head office and branch also the expansion of external and internal trade and to provide quality of work, smooth communication between employees and create an appropriate work environment which contributes to raise the work efficiency.

\section{Problem definition}

The problem in the slow speed of the internet is, when most of the employees accessing to the internet at same time (High utilization) and this affect their business and the quality of work, also the company branch can't take advantage of the centralized servers service (existing ADS \& File servers) because the ADSL does not support the connection between branches and so it doesn't have stable bandwidth. The company head office had difficulties to manage all wireless access point at same time, they have to do configuration for each as a separate link which take more time for installation and troubleshooting due to slow speed internet.
The company head office had difficulties to manage all wireless access point at same time, they have to do configuration for each as a separate link which take more time for installation and troubleshooting due to slow speed internet. Also they need to reduce monthly phone bills payment which affects the company head office profits and they want easy way for their employees to make calls internally by providing each employee with its own extension number using VOIP. In order to enhance company infrastructure they need new technology to improve the quality of the network and to increase company profit by implementing such new technology based connectivity between factories network infrastructure for stability and scalability of the link in the work environment, which was considered as a critical need to raise the better work efficiency.

\section{Literature review}

Literature review is to look at the scientific article, books and other sources related to a particular topic, theory, summary, the field of research and evaluation of the work, and the aim is to provide an overview of the topic. In the VPN over ADSL scenario, the company connected to the public Internet, which has quite a variation of performance (Haris, 2007). The ISP provides ADSL connectivity with low price than MPLS service in which many organizations can reduce bill payment (Partsenidis, C., 2013). But in MPLS the speed is stable that not depending on other customer's usage.

In MPLS, the configuration of router and core network that happen through ISP engineers and they have full access and

a. Department of Computer Science, Middle East college, Muscat, Oman

Correspondence: indrani@mec.edu.om 
control for MPLS (Garson, S., 2015). If you have internet service with high quality connection, in this case you can use the phone service via the internet instead of using local phone service which provided by the telecom companies (Krishnan, 2014). Wireless Controller simplifies the network deployment, management and operations, because it reduces the overall operational costs of the organization (Zhang, Z., 2015). Firewall is a network protection device that can control and monitor the outgoing and incoming the network traffic according to the security rules specified in the former (Joffe, 2016). The greatest attractive feature which explained by (Cindy, 2013) is Unified Messaging This feature can make users ability MS exchange Server as a voicemail server to reduce the telephone cost.

\section{Methodology}

Methodology is a system of the general precepts or the rules that from which a specific procedures or methods can be derived to understand or solve the different teething troubles in the interior of scope for a certain discipline. The reason of choosing the PPDIOO Methodology as shown in "Fig. 1," This method helps to reduce the total cost of the ownership network and it can also make changes in the plan of infrastructure and resource requirement in the correct manner. And also in addition to that, I want to use a strong and solid design of the network and ascertains well from network operations. PPDIOO will help me to improve the availability of the network and also provides speed in access to the network applications and resources. PPDIOO is an acronym for the six words (Prepare, Plan, Design, Implement, Operate and Optimize).

- Prepare stage: In general, in the Preparation stage, the company will benefit to develop its business requirements, as well as strategy and vision in terms of technology. It is extremely significant to started with this stage to make sure that the network has developed according to the business requirements as much as possible. The output of this stage includes:

\section{1- HLD (High Level Design)}

\section{2- CRD (Customer Requirement Documents)}

\section{3- CSAS (Current State Assessment Survey)}

- Plan stage: It's based on the Preparation stage, use the planning stage based on the company's existing network state. If you don't have any current network, it should be a project plan that include a collection of the required information's before starting the next stage, information includes the allocation of financial and physical resources for the design and implementation of all stages, and develop a plan for the security of the network and the project schedule to fit with the business requirements that are found in the preparation stage. The output of this stage includes:

1- SRS (Site Requirements Specifications)

2- STP (Solutions Test Plan)

3- SSF (Site Survey Form)

4- CRDR (Customer Requirements Document Response)

- Design stage: Through the design stage, the company is updating or developing an integrated network design, it is an important to use all the information collected during the first and second stages to make sure that this design covers all technical and business requirements. If you have been completed everything correctly, it means that the design is capable of providing a network manages the functions required daily, and meets or exceeds all expected reliability, availability, security, scalability and performance standards. The output of this stage include:

\section{1- LLD (Low Level Design)}

- Implement stage: There are different ways can be used to implement, and it's good to use the virtual machine for test to simulate some stages of network design. Using this way allows implementers to find potential problems, and if you found problems, it should resolve in the virtual machine before continuing with full implementation process.

There could be logistical problems beyond the framework of simulators, for example, you must determine who is responsible for the deployment and configuration, testing and operation of the network through the various stages. The output of this stage includes:

\section{1- NRFU Test (Network Ready For Use)}

\section{2- NRFU Testing Report.}

\section{3- IL (Implementation Log)}

- Operate stage: Operate stage is one of the longest of PPDIOO stages, at this stage the company spending money to manage the network which includes reactive and proactive monitoring, trouble management, performance management, security management, and also ability to monitoring and planning. Any minor additions, moves or change the MAC occur in cases at this stage.

The output of this stage includes:

1- RCAR (Root Cause Analysis Reports)

2- MAC Reports

\section{3- SMART net (Support Contract Analysis)}

- Optimize stage: Optimize stage can occur after the operation of the network, this happens if there is any slight or considerable change in the technical requirements for the network or in the business of the company.

During this stage, will be compared to the current technical requirements and business with those used in beginning when the network was designed, if there is any need to make change, then all the stages will start from the beginning to ensure perfect design

Depends on the existing company network as shown in the "Fig. 2," , this project will help to enhance the existing company network which it gives direct impact in improving the quality of work using the proposed network design. 


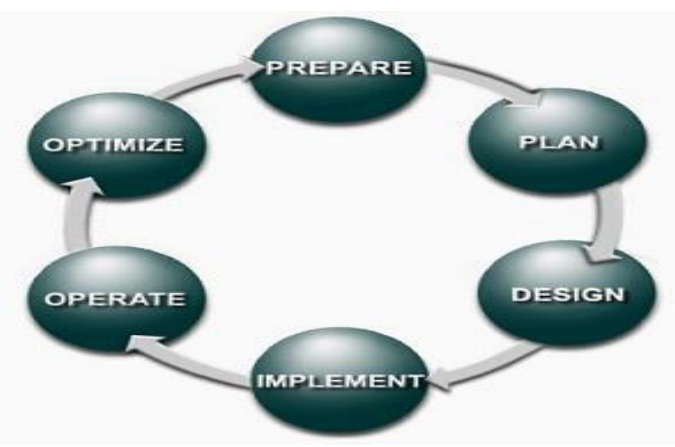

Fig. 1" PPDIOO Model"

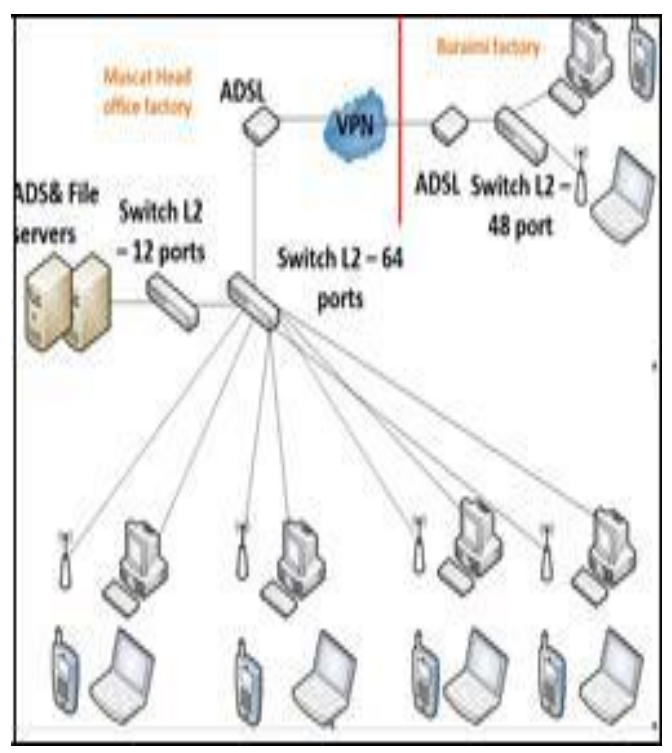

Fig.2" Existing network design"

\section{Proposed system}

The proposed system is making the company communication stable and fast data exchanges between head office factory and branch factory as shown in the Fig. 3.

MPLS line is significant to make genuine connectivity between branches, also provides the employee fast and stable access to the network without delay of data connections among them, in this situation we can build centralized servers in head office factory as a central data management.

\section{Conclusion}

Depends on the existing company network issues, this project will help to enhance the existing company network which its direct impact in improving the quality of work, fast communication between head office and branch also the expansion of external and internal trade. Using MPLS connectivity between head office factory and branch factory to provide stability and scalability of link and also able to build servers for exchange the services between branches. For more security of the network for head office and branch, the Firewall is very important to secure the company network from external and internal threats. Using wireless controller in head office, it can manage all access point at same time.

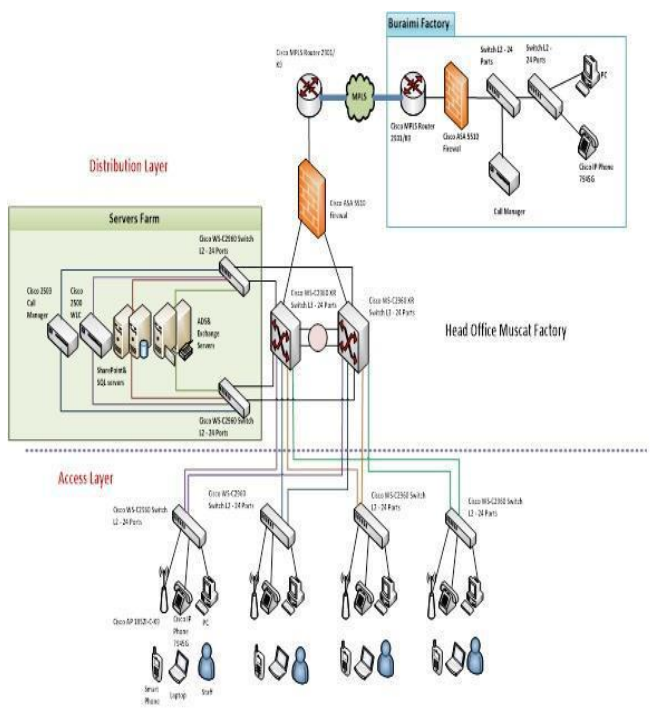

Fig.3 "Proposed Network design Infrastructure"

Migration to MPLS connectivity between head office and branch office can provide more stability and scalability communication

\section{Acknowledgement}

I extend my sincere thanks and gratitude to all those who helped me and gave me advice and guidance and special thanks to my supervisor Ms. Indrani P, for standing beside me by giving the necessary instructions and guidance.

\section{References}

Haris, (2007). www.supportforums.cisco.com. [Online] https://supportforums.cisco.com/discussion/10328246/mp ls-vpn-over-dsl, [Accessed 27 October 2016]

Partsenidis, C., (2013). www.teachtarget.com. [Online] http://searchenterprisewan.techtarget.com/tutorial/MPLSvs-VPN-Comparing-ADSL-MPLS-VPNs-with-ADSL-IPVPNs, [Accessed 26 October 2016]

Garson, S., (2015). www.sd-wan-experts.com. [Online] https://www.sd-wan-experts.com/wan-using-ip-vpn-overinternet-versus-mpls-pros-and-cons/, [Accessed 26 October 2016].

Krishnan, (2014). www.freepatentsonline.com. [Online] http://www.freepatentsonline.com/y2016/0105566.html, [Accessed 3 November 2016].

Zhang, Z., (2015). www.google.com. [Online] http://www.google.com/patents/US20160278001, [Accessed 9 November 2016].

Joffe, R. L., (2016). www.freepatentsonline.com. [Online] http://www.freepatentsonline.com/9356942.html, [Accessed 13 November 2016]

Cindy, (2013). http://office.about.com. [Online] http://office.about.com/od/Office365/a/Summary-OfFeatures-In-Microsoft-Exchange-Server-2013.htm, [Accessed 29 October 2016] 